\title{
Colección de Mamíferos Corporación Universitaria Santa Rosa de Cabal (CUS-M)
}

\author{
Castaño Salazar, John Harold ${ }^{1 *(i)}$ \\ 1 Corporación Universitaria Santa Rosa de Cabal - UNISARC, Vereda el Jazmín, Km 4 Santa Rosa de Cabal, Risaralda \\ * Correspondencia: john.castano@unisarc.edu.co
}

\begin{abstract}
Resumen
La colección de mamíferos de la Corporación Universitaria Santa Rosa de Cabal (CUS-M) está ubicada Santa Rosa de Cabal, Risaralda. Alberga 503 especímenes pertenecientes a 10 órdenes, 27 familias, 75 géneros y 99 especies que corresponden al 78 \% de las especies registradas para el departamento de Risaralda y al $19 \%$ de las especies registradas para Colombia. De los especímenes que preserva la colección el $81 \%$ corresponde a pieles y esqueletos en seco, $13 \%$ en líquido y $6 \%$ huellas en yeso. Adicional a esto, se cuenta con una colección de referencia de semillas de la dieta de mamíferos frugivoros. Los especímenes provienen de los departamentos de Risaralda (467), Caldas (5), Cundinamarca (3) y Valle del Cauca (2), de alrededor de 100 localidades distribuidas en 14 municipios.
\end{abstract}

Palabras clave: Andes del Norte, Chocó Biogeográfico, Eje Cafetero, Especímenes, Paisaje Cultural Cafetero, Risaralda.

\section{Abstract}

The collection of mammals of the Santa Rosa de Cabal University Corporation (CUS-M) is located in Santa Rosa de Cabal, Risaralda. It houses 503 specimens belonging to 10 orders, 27 families, 75 genera and 99 species, corresponding to $78 \%$ of the species registered for the department of Risaralda and $19 \%$ of the species registered for Colombia. Of the specimens preserved by the collection, $81 \%$ correspond to skins and skeletons dry, $13 \%$ in liquid and $6 \%$ traces in plaster. In addition to this, there is a reference collection of seeds from the diet of frugivorous mammals. The specimens come from the departments of Risaralda (467), Caldas (5), Cundinamarca (3) and Valle del Cauca (2), from around 100 localities distributed in 14 municipalities.

Key words: Northern Andes, Choco, Coffee axis, specimens, coffee cultural landscape.

La colección de mamíferos de la Corporación Universitaria Santa Rosa de Cabal (CUS-M) está ubicada en el campus de la Corporación Universitaria Santa Rosa de Cabal (UNISARC) en la vereda el Jazmín, Km 4 Santa Rosa de Cabal, Risaralda. La colección se originó en el año 2012 como una iniciativa docente y del semillero de investigación en mastozoología de UNISARC, con el fin de preservar material de referencia del departamento de Risaralda. En la actualidad, la colección alberga 503 especímenes de mamíferos pertenecientes a 10 
órdenes, 27 familias, 75 géneros y 99 especies (Tabla 1). Los órdenes mejor representados son Chiroptera y Rodentia con 54 y 21 especies, respectivamente (Tabla 1).

Tabla 1. Órdenes, familias, géneros y especies de mamíferos depositados en la Colección de Mamíferos Corporación Universitaria Santa Rosa de Cabal (CUS-M).

\begin{tabular}{lcccc}
\hline Orden & Familias & Géneros & Especies & Número de especímenes \\
\hline Didelphimorphia & 1 & 7 & 8 & 29 \\
Paucituberculata & 1 & 1 & 1 & 1 \\
Cingulata & 1 & 1 & 1 & 4 \\
Pilosa & 1 & 1 & 1 & 1 \\
Chiroptera & 5 & 32 & 54 & 356 \\
Carnivora & 4 & 9 & 9 & 34 \\
Perissodactyla & 1 & 1 & 1 & 2 \\
Artiodactyla & 1 & 1 & 2 & 2 \\
Rodentia & 9 & 19 & 21 & 68 \\
Lagomorpha & 1 & 1 & 1 & 4 \\
\hline Total & 25 & 73 & 99 & 501 \\
\hline
\end{tabular}

De los especímenes preservados en la colección, el 81 \% corresponde a pieles y esqueletos en seco, $13 \%$ en líquido y $6 \%$ huellas en yeso. Adicional a esto, se cuenta con una colección de referencia de semillas de la dieta de mamíferos frugivoros. Los especímenes provienen de los departamentos de Risaralda (467 especímenes), Caldas (5 especimenes), Cundinamarca (3 especimenes) y Valle del Cauca (2 especimenes), y cerca de 100 localidades distribuidas en 14 municipios concentrados principalmente en el Paisaje Cultural Cafetero y el Chocó biogeográfico. Un total de 504 especímenes están identificados a nivel de clase, 504 a orden, 500 a familia o subfamilia, 491 a género, 476 a especie y 7 a subespecie. En la colección están representadas 99 especies que corresponden al 78 \% de las especies registradas para el departamento de Risaralda (Castaño et al., 2018) y al 19 \% de las especies registradas para Colombia (Ramírez-Chaves et al., 2019).

Los especímenes de la colección de mamíferos CUS-M han sido empleados en al menos 6 publicaciones científicas (Torres, et al., 2014; Castaño \& Carranza-Quiceno, 2015; CarranzaQuiceno et al., 2018; Castaño, et al., 2018; Castaño, et al., 2020; Castaño \& Hernández, 2020) y dos más en preparación.

La información de la colección de mamíferos CUS-M, cuenta con información pública y disponible a través de solicitud al correo electrónico: john.castano@unisarc.edu.co. Recientemente se inició la revisión de datos y la actualización taxonómica de los especímenes de la colección, con el fin de garantizar una mejor calidad de los datos disponibles.

Entre los principales recolectores en la colección de mamíferos CUS-M se encuentran John Harold Castaño (85 especímenes), Mariana Vélez (84 especímenes), Diego Alejandro Torres (75 especímenes), entre otros.

\section{AGRADECIMIENTOS}

A los participantes del semillero de investigación en mamíferos de UNISARC SIMAZU por su valioso trabajo y constante contribución al desarrollo y mantenimiento de la colección, especialmente a Luis Fernando Ruíz, Esteban Grajales y Diego Torres. 


\section{REFERENCIAS}

Carranza-Quiceno JA, González G, Tapasco DY, Cortes-Bedoya S, Mantilla JC, Pareja IM, Payan JE, Montoya M, Henao JR, Quintero-García S, López-Barrera AM, Rivera-Gómez J, Cárdenas BA, Maturana MY, Martínez A, Machado Y, Castaño JH, Muñoz LH. (2018). Biodiversidad en el área de manejo especial étnico alto Amurrupá, Santa Cecilia, Risaralda. En Carder, IIAP, PNNC, \& Unisarc (Eds.), Diveridad Biológica y Cultural del Pacífico Risaraldense (pp. 147-184). Pereira, Colombia: IIAP, Carder, Unisarc.

Castaño JH \& Carranza-Quiceno JA. (2015). Campoalegre biodiversidad en un paisaje Rural Andino de Riaralda. (J. H. Castaño \& J. A. Carranza-Quiceno, Eds.). Santa Rosa de Cabal: Corporación Autónoma Regional de Risaralda - CARDER, Universidad de Santa Rosa de Cabal - UNISARC.

Castaño JH, Carranza-Quiceno JA \& Pérez-Torres J. (2018). Diet and trophic structure in assemblages of montane frugivorous phyllostomid bats. Acta Oecologica, 91, 81-90. https:// doi.org/10.1016/j.actao.2018.06.005

Castaño JH, Carranza-Quiceno JA, \& Pérez-Torres J. (2020). Bat-fruit networks structure resist habitat modification but species roles change in the most transformed habitats. Acta Oecologica, 105(9), 103550. https:// doi.org/https:// doi.org/10.1016/j.actao.2020.103550

Castaño JH \& Hernández A. (2020). Mamíferos silvestres del área metropolitana PereiraDosquebradas. En UTP (Ed.), La Investigación un proceso de alianzas para el desarrollo regional (pp. 311-351). UTP.

Castaño JH, Torres DA, Rojas-Díaz V, Saavedra-Rodríguez CA \& Pérez-Torres, J. (2018). Mamíferos del departamento de Risaralda, Colombia. Biota Colombiana, 18(2), 239-254. https:// doi.org/10.21068/BC.V1812.505

Ramírez-Chaves HE, Suárez Castro AF, Zurc D, Concha Osbahr DC, Trujillo A, Noguera Urbano EA, Pantoja Peña GE, Rodríguez Posada ME, González Maya JF, Pérez Torres J, Mantilla Meluk H, López Castañeda C, Velásquez Valencia A, Zárrate Charry D. (2019). Lista de referencia de especies de mamíferos de Colombia. Versión 1.2. https://doi.org/10.15472/kl1whs

Torres DA, Henao JR \& Castaño JH. (2014). Primer registro de Anoura cultrata (Chiroptera: Phyllostomidae) para la cuenca del río Cauca, Colombia. Mammalogy Notes, 1(2), 9-11.

Editor: Miguel E. Rodríguez-Posada

Recibido 2020-04-16

Revisado 2020-04-21

Aceptado 2020-05-13

Publicado 2020-06-12 